\title{
Antibiotic apocalypse; are we ready?
}

\section{Editorial}

Look to the past is the way to discover our future. A paper-cut wound, any kind of surgery, giving birth, and a simple infection could kill you in the past before the antibiotic (pre-antibiotic era) and it will kill you in the future after the antibiotic (antibiotic apocalypse or postantibiotic era). The Magic Word - Antibiotic unavoidably leaping to our mind when we dealing with any infection. Since 1928 mould metabolites (e.g. Penicillin) has been widely used to treat bacterial infection, although the medical use of the mould is not new. The ancient Egyptians were leaders in using the mould in the treatment of infection as mentioned in the landmark book "Odyssey". The book was written by Homer who considered as one of the most famous writers in the history of the world. Homer mention that, the ancient Egyptian men were the most skillful human in the medicine. Some of their wound healing practices are still used till now with exalt of their medicinal heritage. Imhotep (2650-2600 BC); the remarkable ancient Egyptian doctor is known to treat infection with mouldy bread. This fact was proven in September 1928, by the Scottish physician Alexander Fleming. During his work at St. Mary's Hospital in London as bacteriologist, Alexander Fleming was noticed an interesting phenomenon. The grown colonies of the bacterium Staphylococcus aureus appeared translucent: "ghostly," in Fleming's words. He discovered that the reason of change of the bacterial colonies growth from the usual yellow, opaque mass, to translucent: "ghostly," was the contamination of the Petri dish with a fungus. The fungus contaminating the Petri dish was eventually identified as Penicillium notatum, and Fleming termed the lytic compound produced by this mold, "penicillin". Clinical application of the penicillin was done by Florey in 1940. Due to his pioneer work, Alexander Fleming, who with colleagues won the Nobel Prize (1945) in Physiology and Medicine, said in his Nobel lecture: 'development of the bacterial resistance to the penicillin in the laboratory by exposing them to concentrations not sufficient to kill them. Years ago, the World Health Organization began warning about the 'post antibiotic era'a time when drug resistance prevails, and antibiotics are no longer effective. The reality is, for many people, that era is already here. U.S. health officials on Thursday (May 26, 2016) reported the first case in the country of a patient with an infection resistant to a last-resort antibiotic, and expressed critical fear that the microorganism could pose serious threat for routine infections if it spreads. Referring to the urinary tract infection of a 49-year-old female patient who had unable to travel for five months. Thomas Frieden, director of the U.S. Centers for Disease Control and Prevention, said "the risk of post-antibiotic world already exist," The bacteria were resistant to colistin, an antibiotic that is reserved for use against "terrifying bacteria". Antibiotic apocalypse, are we ready? People will die from bacterial pneumonia, wounds, infections, sepsis and bacteraemia. The role of Doctors, nurses and clinicians will be limited to duty of care or ablate of the infected tissue. The healthcare system will be overloaded and most probably will collapse. We will stand useless watching the devastating consequences of demise of one of our most precious medical cures. So, I think we are not ready. Development of strategic plans based on epidemiologic studies of antibiotic use and resistance is a must. The overall uptake of antibiotics in a population,
Volume 5 Issue 3 - 2016

\author{
Ayman Hegab \\ Clinical Associate Professor of Oral \& Maxillofacial Surgery, \\ Al-Azhar University, Egypt
}

Correspondence: Ayman Hegab, Clinical Associate Professor of Oral \& Maxillofacial Surgery, Faculty of Dental Medicine, Al-Azhar University, Cairo, Egypt, Tel 00974 33310124, Fax 201000000000,Email hegab@mail.com

Received: October 27, 2016 | Published: October 31, 2016

as well as how antibiotics are consumed, has an influence on antibiotic resistance. Perhaps the most important is the role played by medical and dental professionals. The use of antibiotics for the treatment of various conditions has been on the rise for past few decades. ${ }^{1}$ Growing evidence that a significant amount of antibiotic consumption based on unsuitable or improper prescriptions, giving us a red alarm. For example, in the USA, a fifth of the total antibiotic prescription was found to be for the treatment of upper respiratory tract infections, even though these infections are almost always viral. ${ }^{2}$ Dentists depend on the antibiotics for the treatment of a widespread variety of odontogenic and non-odontogenic conditions. It is, however, important to note that $6-10 \%$ of all antibiotic prescriptions have been found to be made by dentists. With evidence suggesting that a lot of prescriptions made by dentists might be on empirical basis, and not supported by scientific knowledge, this small percentage of the total antibiotic prescription can play a significant role in the development of antibiotic resistance. ${ }^{3}$ The factors affecting prescription behavior are complex and interlinked. Drug prescribing habits have found to be effected by the practitioner's level of knowledge, patient's knowledge or lack of cultural beliefs, past experiences, economic factors and use of diagnostic aides such as microbial testing. Misuse and over prescription of antibiotics is considered to be the most important cause in the development of antibiotic resistance. ${ }^{4,5}$ Antibiotics are prescribed indiscriminately, inappropriately and injudiciously to manage the oral diseases. Dental diseases are predominantly because of local factors; then elimination of the local contributing factors minimizes the need for prescribing antibiotics considerably. Despite the awareness on antibiotic resistance, dentists show lack of concern in restriction of antibiotic prescriptions which represent serious public health problem. The art and science of using the antibiotic is the key of success to prevent the Antibiotic apocalypse. Principles for Prescribing Antibiotics by dentists include; Infections are cured by the host not by the antibiotics. Antibiotics are not an alternative to dental intervention, removal of the local causal factors decreases the necessity for the use of antibiotics. Medical interferences, for example incision and drainage, extraction, or endodontic therapy, are appropriate first stages in the management of most oral infections. Consider the potential benefits and risks of antibiotics before prescribing. Prescribe antibiotic in case of clinical indications only. 
Prescribe antibiotic when risk/benefit balance is favorable. It is not a substitute for establishing adequate drainage. Choose the effective narrowest spectrum antibiotic. Prescribe an adequate dose. Prescribe for appropriate period. Choose the antibiotic having least side effects. Consider antibiotic sensitivity tests. Recognize that antibiotics encourage development of resistance if used for too long and/or at sub-optimal doses. Consider replacements of the systemic antibiotics with local debridement and application of topical antiseptics. Oral viral infections, fungal infections, or oral ulcerations related to trauma or aphthae are not indications for antibiotic prescriptions.

\section{Funding}

None.

\section{Acknowledgments}

None.

\section{Conflicts of interest}

Author declares that there is no conflict of interest.

\section{References}

1. Preus HR, Albandar JM, Gjermo P. Antibiotic prescribing practices among Norwegian dentists. J Dent Res. 1992;100(4):232-235.

2. Gonzales R, Steiner JF, Sande MA. Antibiotic prescribing for adults with colds, upper respiratory tract infections, and bronchitis by ambulatory care physicians. JAMA. 1997;278(11):901-904.

3. Roda RP, Bagan JV, Bielsa JMS, et al. Antibiotic use in dental practice: a review. Med Oral Patol Oral Cir Bucal. 2007;12(3):E186-E192.

4. Mac Farlene J, Holmes W, Mac Farlene R, et al. Influence of patients' expectation on antibiotic management of acute lower respiratory tract illness in general practice: a questionnaire study. BMJ. 1997;315(7117):1211-1214.

5. Bisno AL, Gerber MA, Gwaltney JM, et al. Diagnosis and management of group A streptococci pharyngitis: a practice guideline. Clin Infect Dis. 1997;25:574-583. 